\title{
An illusion of eccentricity
}

\author{
SERGIO RONCATO \\ Università di Padova, Padova, Italy
}

\begin{abstract}
The illusion investigated here is that two concentric arcs, drawn in different (though possibly overlapping) circular sectors and having the same angular extent, appear to be eccentric. Three possible explanations of the illusion are tested. The first hypothesis is that concentricity judg. ments are made by elongating the arce to see if they intersect, the illusion being due to the tendency, when elongating a curve, to follow the end-tangent. The second hypothesis is that concentricity judgments are based on a test of coincidence of centers, the illusion being due to the overestimation of the radius of short arcs. The third hypothesis is that both of these factors contribute in equal measure. These hypotheses make different predictions about the effect (on the magnitude of the illusion) of the following variables: (1) the angular distance between the arcs; (2) the radial distance between the arcs; (3) the degree of curvature of the arcs; and (4) the angular extent of the arcs. The observed values of the illusion angle (obtained by the method of limits) in relation to these variables did not uniformly support any of the hypotheses. A more complex model that is consistent with the observed results is therefore proposed.
\end{abstract}

The purpose of this paper is to report and examine some possible explanations of what will be called the "eccentricity illusion." This illusion appears when concentric arcs of the same angular extent are drawn in different circular sectors: the arcs, although in fact concentric, appear to converge in the direction of where the extension of their chords would intersect (see Figure 3). The effect is illustrated by the three configurations in Figure 1. Each configuration consists of three arcs which are concentric about the point indicated. In Figure 1a, the arcs occupy the same circular sector and appear to be concentric. In Figure $1 \mathrm{~b}$, the central axis of the lowest arc is rotated clockwise by 20 deg with respect to the central axes of the other two arcs; the result is that the lowest arc appears to converge with the upper arcs on the lefthand side of the figure, the side where the chords would intersect if extended. In Figure 1c, the arcs are drawn in three different sectors and no two of them appear to be concentric; rather, they appear to converge on the left-hand side near the point where their chords would intersect if extended. The illusion is demonstrated more dramatically in the two configurations in Figure 2; these are composed in such a way as to highlight the contrast between arcs that cover the same circular sector and arcs that cover overlapping, but different, sectors.

This research was supported by grants of the Ministero Pubblica Istruzione and Consiglio Nazionale delle Ricerche n. 8100074.04. Thanks are extended to G. Vicario, P. Bozzi, G. Kanisza, P. Legrenzi, M. Sambin, R. Luccio, W. Gerbino, and A. Bazzeo for useful comments on an earlier draft of this paper and to $\mathbf{R}$. Power for his revision of the English test and for several helpful criticisms. The author's address is: Istituto di Psicologia, Università di Padova, Piazza Capitaniato, 5, 35100 Padova, Italia.
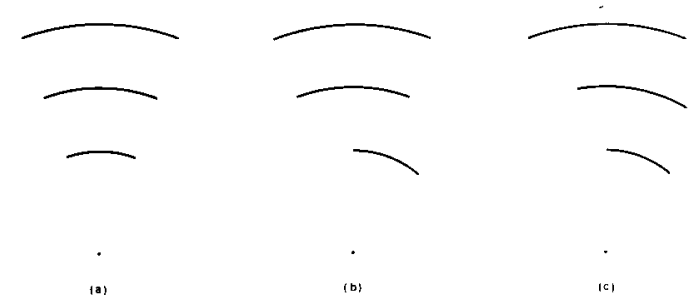

Figure 1. Illustration of eccentricity lllusion. All the three configurations are made of concentric arcs, although (b) and (c) do not appear to be. The black dots represent the common centers of the curves.
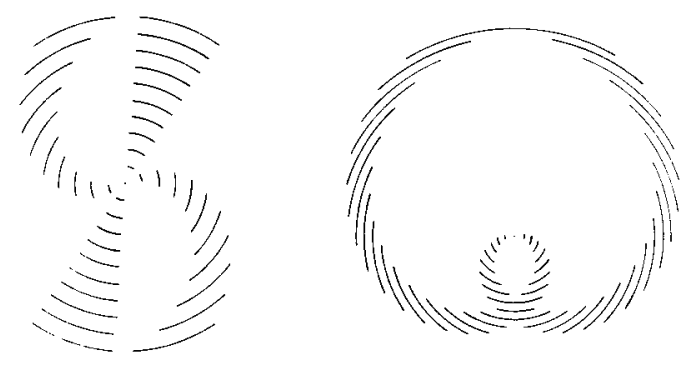
Filgur.

Igure 2. Two more configurations showing an eccentricity illu-

The experiment reported here examines the effect of four variables on the magnitude of the illusion; the variables are (1) the angle of intersection of the chords, (2) the distance between the arcs, (3) the angular extent of the arc sectors, and (4) the curvature of the arcs. These variables were examined with the aim of testing three possible explanations of the illusion, which will be described shortly. First, however, a preliminary question should be discussed: Is the eccentricity illusion an independent phenomenon, 
or is it merely a special case of some other illusion that has already been reported?

One possibility that should be considered is whether the eccentricity illusion can be reduced to the well-known illusion of the flattening of short arcs (Tolansky, 1964). In Figures $1 \mathrm{~b}$ and $1 \mathrm{c}$, for example, it could be argued that the curvature of the lowest arcs will be underestimated (relative to that of the upper arcs) and that the arcs in each configuration will, as a result, appear eccentric. But this fails to explain why the eccentricity illusion does not also occur in Figure 1a; if the illusion is merely due to the flattening effect, it should occur in equal fashion when the arcs cover the same sector. The eccentricity illusion thus cannot be reduced to the flattening illusion.

Another illusion that is superficially similar to the eccentricity illusion is Fraser's "twisted chord" figure, in which concentric circles are perceived as a spiral. However, the two illusions are not comparable, since the circles in the Fraser illusion are composed not of concentric arcs, but sections of spirals. Consequently, this attempt to reduce the eccentricity illusion to another more familiar illusion also fails.

How, then, can the eccentricity illusion be explained? It will be assumed from now on that two "distorting mechanisms" are likely to be relevant: first, the tendency to veer towards the end tangent when attempting to mentally elongate a curve (Virsu, 1971a), and, second, the tendency to underestimate the curvature of short arcs and consequently to misperceive their centers (Virsu, 1971a, 1971b). Three hypotheses therefore need to be considered: the il- lusion may be due to (1) the first distorting mechanism only, (2) the second mechanism only, or (3) some combination of the two.

Before examining these hypotheses in detail, it will be useful to establish some terminology. First of all, the angle between the central axes of two concentric arcs (the angle $K \widehat{R} H$, marked $\alpha$ in Figure $3 a$ ) will be referred to as the "central axis angle" (this angle is equal to the angle of intersection of the end tangents of the arcs-BPD in Figure 3a-and also the angle of the intersection of their chords). Next, the angular extent of an arc-in other words the angle formed by the radii from the ends of the arc-will be called the "center angle" of the arc. Thus, the center angle of the arc $\widehat{C D}$ in Figure $3 a$ is $C \widehat{R D}$, and has the value $\gamma$. Finally, it is useful to have some measure of the extent of the illusion, of the difference between the real concentricity and the phenomenal concentricity. This may be done by imagining a situation in which a perceiver is presented with two concentric arcs covering different sectors (see $\widehat{\mathrm{AB}}$ and $\widehat{\mathrm{CD}}$ in Figure 3a) and asked to rotate $\overparen{C D}$ about $K$ until the two arcs appear to be parallel (concentric). The resulting angle of rotation, marked $\beta$ in Figure $3 b$, serves as a measure of the eccentricity illusion and will be referred to as the "illusion angle."

The hypotheses listed above will now be examined in turn.

\section{The End-Tangent Hypothesis}

The task of judging whether two arcs are concentric would intuitively seem to be performed by the
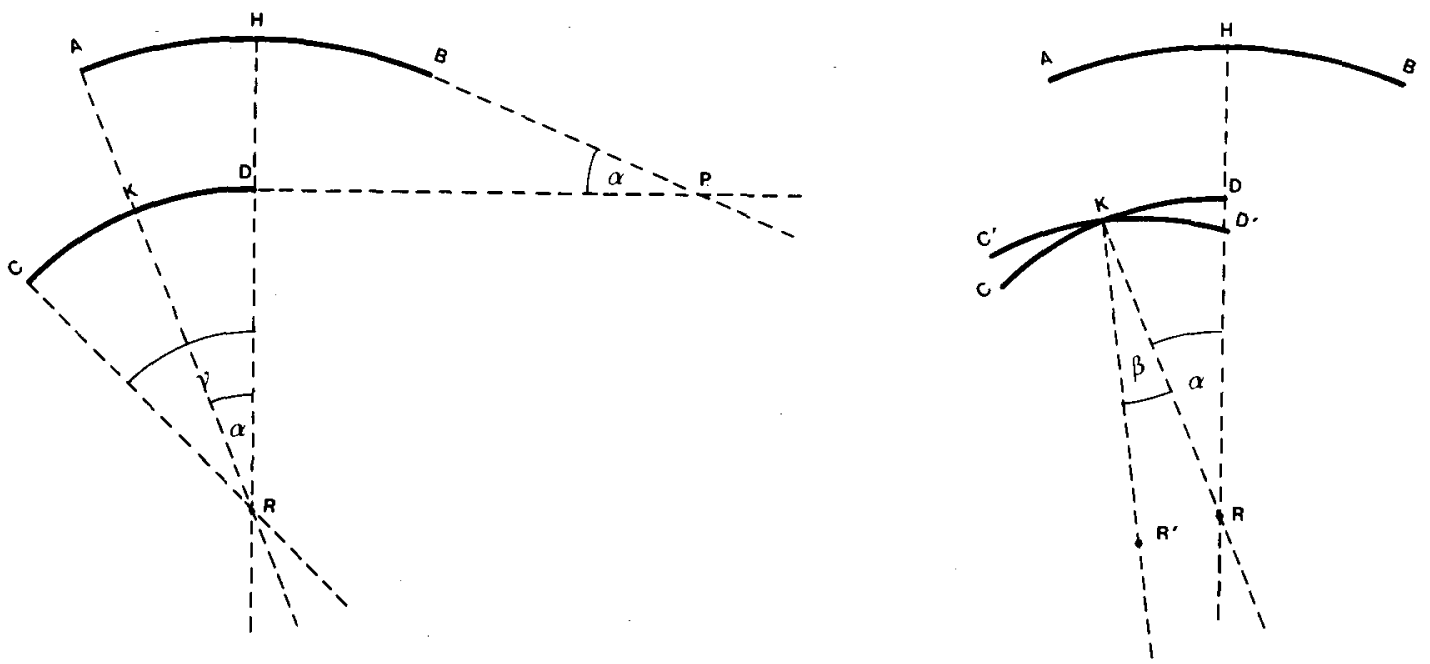

(a)

(b)

Figure 3. (a) Solid lines show a pair of concentric arcs which served as stimuli in the present experiment. Dashed lines have been drawn to represent tangents, radii, and central axes. The angle $\alpha$ intercepted by the two axes RH and RK is $20 \mathrm{deg}$; the center angie $\gamma$ is $40 \mathrm{deg}$. (b) The method for estimating the amount of eccentricity illusion is schematized: the arc $\overparen{\mathrm{CD}}$ can be rotated about $\mathrm{K}$ until it appears parallel to $\widehat{\mathrm{AB}}$. $R$ indicates the common center of

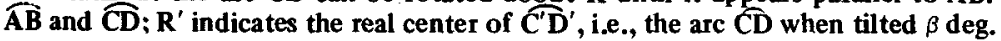


strategy of mentally elongating the arcs and finding out whether or not they cross. In fact, in an earlier study of the illusion (Roncato, Note 1), many of the subjects reported using this strategy. If such elongation of the arcs is used, a plausible explanation can be found in the phenomenon of "tangent escape." Virsu (1971a) has shown that the postulation of this distortion mechanism- a tendency to follow the end tangent when attempting to mentally elongate a curve-can account well for a misperception of curvature and of arc intersection. The evidence for this distortion mechanism includes Virsu's (1971a) finding that subjects tend to perform rectilinear eye movements when perceiving a curve and studies (Crassini \& Over, 1975; Timney \& McDonald, 1978) that have shown that arcs are processed by the eye, at least in part, as a series of tangential segments.

A possible hypothesis, then, is that judgments of concentricity are based on a test of whether the end tangents of the arcs are parallel. This strategy would give the correct answer only when the arcs covered the same circular sector; if, instead, the arcs covered different sectors, the strategy would give the wrong answer, since the end tangents of two concentric arcs intersect in different sectors. Assuming that the center angles of the arcs are equal, the angle of intersection of the end tangents will be equal to the central angle (see Figure $3 \mathrm{a}$ ). The hypothesis therefore predicts that the extent of the illusion (i.e., the illusion angle) will be equal to the central axis angle (i.e., that in Figure $3 b, \beta=\alpha$ ), since it is a rotation of this magnitude that is required in order to make the end tangents of the arcs parallel. (It should be noted, by the way, that this prediction also follows from the alternative hypothesis that concentricity judgments are based on a comparison of the chords of the arcs rather than of the end tangents.)

\section{The Centers-Attraction Hypothesis}

This hypothesis is based on the phenomenon, demonstrated by Virsu (1971a, 1971b), that the curvature of a short arc is underestimated (and the radius of the arc, correspondingly overestimated) by an amount proportional to the length of the arc. The way in which this phenomenon might explain the eccentricity illusion is shown in Figure 4 . In Figure $4 a$, the arcs 1 and 2 are concentric, so that the real centers $R_{1}$ and $R_{2}$ coincide. The apparent centers $A_{1}$ and $A_{2}$, which have been drawn according to Virsu's data, lie beyond the real centers, $A_{2}$ being beyond $A_{1}$, since arc 2 is shorter than arc 1 . In Figure $4 b$, the lower arc has been rotated anticlockwise about the real center, $\mathbf{R}$, with the result that the perceived center $\mathbf{A}_{\mathbf{2}}$ is displaced to the right of $A_{1}$; this relationship between the two perceived centers corresponds to the relationship between the two real centers of two eccentric arcs (see Figure 4c). Misperception of the arc centers may therefore explain why the arcs in Figure $4 \mathrm{~b}$ appear to be eccentric.

If this is so, it should be possible to predict the extent of the illusion, using Virsu's calculations, as a function of the radii and angular extent of the arcs. If, when asked to rotate arc 2 (Figure $4 \mathrm{~b}$ ) about $K$ until it is concentric with arc 1 , the subject chooses the orientation that minimizes the distance between the apparent centers, the illusion angle is given by:

$$
\beta=\operatorname{arctg}\left[\sin \alpha(\mathrm{r} / \mathrm{d}+\cos \alpha)^{-1}\right] \text {, }
$$

where $r$ denotes the real radius of the arc 2 in Figure $4 \mathrm{~b}$ and $\mathrm{d}$ denotes the difference between the real and apparent radii of arc 1 -that is, the distance between $R$ and $A_{1}$. The geometrical proof of this result is given in Appendix 1.

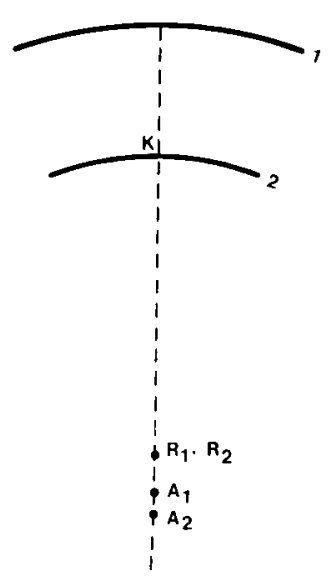

(a)

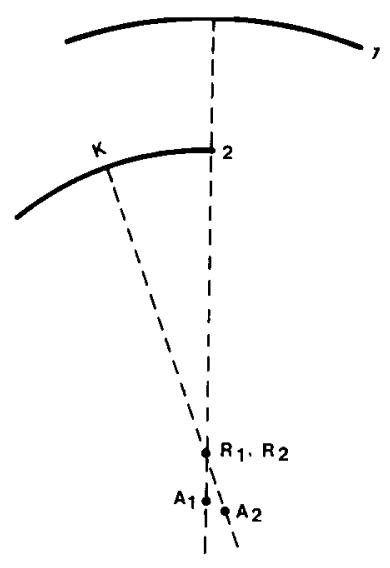

(b)

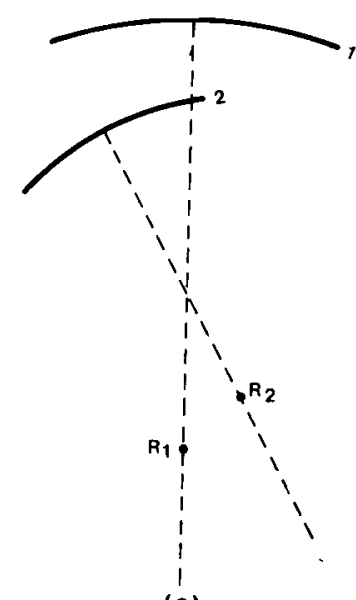

(c)

Figure 4. Dashed lines represent the central axes of the arcs. Dots indicate the centern: $\mathbf{R}_{1}$, $\mathbf{R}_{2}$ are real centers and $A_{1}, A_{2}$ are apparent centers. $K$ indicates the point around which the lower arc can be tlited. 


\section{The A veraging Model}

The next hypothesis that should be considered is that the illusion is due to the combined action of the two distorting mechanisms discussed above. According to this hypothesis, the subject, in trying to judge whether two arcs are concentric, has to balance two criteria: (1) parallelism of end tangents, and (2) minimal distance between apparent centers. The task of balancing these criteria can be most simply understood by imagining again that the subject has been asked to rotate the arc $\widehat{C D}$ (in Figure 3) until it is concentric with $\widehat{\mathrm{AB}}$. At any given value of the rotation angle, each of the two criteria will require that the angle should be left as is, that it should be enlarged, or that it should be reduced. The distortion mechanisms can thus be regarded as tendencies, of varying strengths, to increase or decrease the rotation angle.

The situation is represented schematically in Figure 5a. Different values of the rotation angle (assuming a clockwise rotation given the stimulus of Figure 3a) are displayed on the abscissa, and the tendencies due to the two mechanisms are represented by the vectors, the magnitude of the two vectors (i.e., the strength of the tendency) being indicated on the ordinate. Since, in Figure 3, the arcs are really concentric, the point of real concentricity is represented by a rotation angle of $0 \mathrm{deg}$. The point labeled $D$ is the value of the rotation angle at which the distance between the apparent centers of the arcs is minimal; the point labeled $A$ is the value at which the end tangents are parallel. It is assumed that the strength of a tendency will increase in a linear fashion as the tilt angle diverges from the optimal value, and that the two criteria are given equal weight. The first as- sumption is based on the simple idea that the tendency to adjust the orientation of an object is stronger the more this orientation appears to be distorted; the second assumption merely reflects our inability to decide a priori whether the two tendencies have the same strength or not.

Given these assumptions, it will be seen that the point of balance occurs at $B$, which is midway between $\mathrm{D}$ and $\mathrm{A}$.

Of course, the hypothesis depicted by Figure 5a represents only one of many possible ways in which the two distorting mechanisms might act in combination. It may, in fact, be that the mechanisms have unequal weight: for example, the centers attraction mechanism may outweigh the end-tangent mechanism, as in Figure $5 \mathrm{~b}$, with the result that the balance point $B$ is closer to $D$ than to A. Another, more complex, possibility is that the relative weightings of the two mechanisms vary according to the values of other parameters. For the time being, however, these complications will be ignored, and the only possibility that will be considered is that of Figure 5a, in which the two mechanisms have equal weights.

The three hypotheses just discussed make different predictions about the value of the illusion angle as a function of the four parameters-(a) central axis angle, (b) distance between the arcs, (c) curvature of the arcs, and (d) center angle of the arcs-investigated in the experiment. The end-tangent hypothesis predicts simply that the illusion angle will be equal to the central axis angle, the other three variables having no influence. The averaging hypothesis predicts, in each case, a value of the illusion angle intermediate between the values predicted by the other

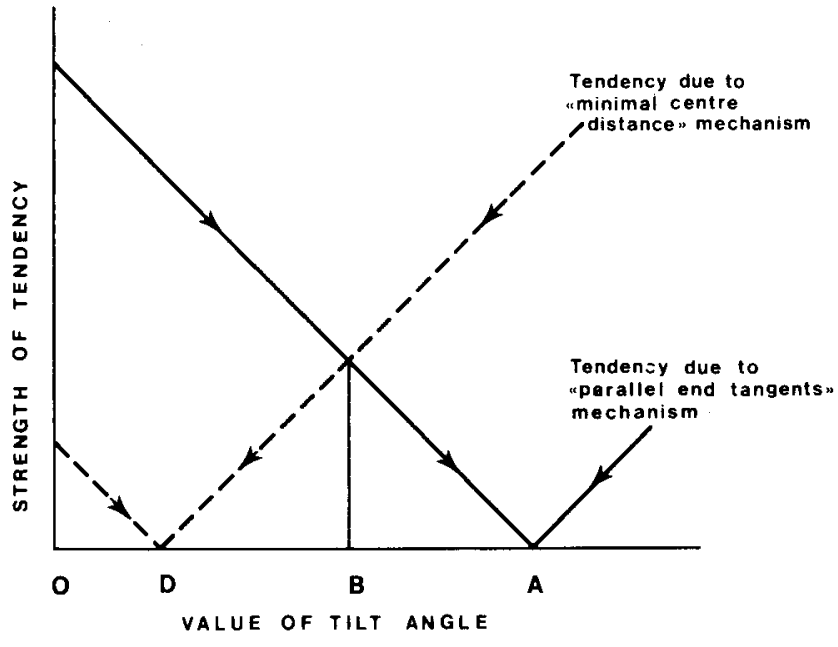

(a)

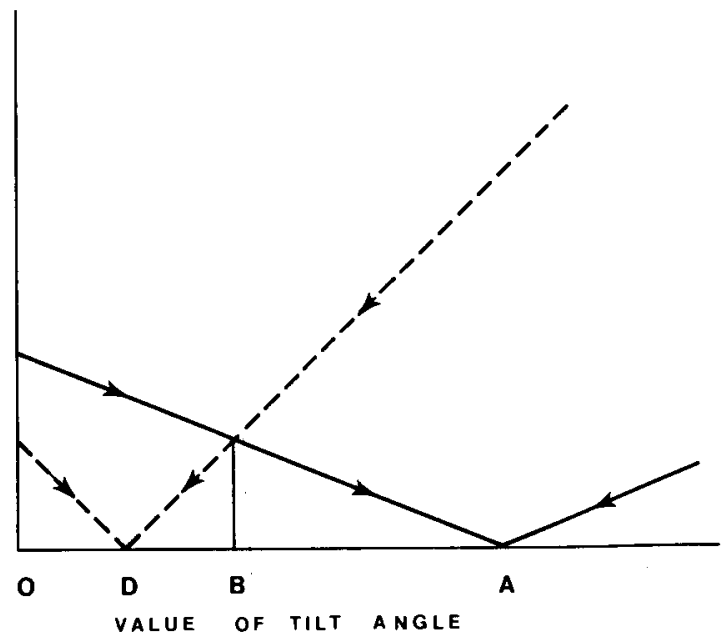

(b)

Figure 5. Gradients representing the strength of the opposing tendencies: end-tangent mechanism (solid line) and centersattraction mechanism (dashed line). See text for details. 
two hypotheses; it therefore requires no independent discussion. The only hypothesis that need be considered in detail is the centers-attraction hypothesis.

\section{Predictions of the Centers-Attraction Hypothesis}

Central axis angle. The centers-attraction hypothesis predicts that the illusion angle will increase as the central angle increases. Unlike the end-tangent hypothesis, it predicts that the illusion angle will be smaller than the central axis angle, not equal to it. From Equation 1, in fact, it can be seen that $\beta<\alpha$. The precise effect of increasing the central axis angle is shown by Figure 6a, which shows an upper arc (1) with real center $R$ and apparent center $A_{1}$, and two lower arcs ( 2 and $2^{\prime}$ ) with real centers at $R$ and apparent centers $A_{2}$ and $A_{2^{\prime}}$. Arcs 2 and $2^{\prime}$ have the same radius, but form different central axis angles with arc 1 , so that arc 2 ' represents the result of increasing the central axis angle while keeping the other variables constant. Now, according to the centersattraction hypothesis, apparent concentricity is obtained when the rotation angle is such as to minimize the distance between the apparent centers; it can be shown (see Appendix 1) that this distance is minimal when the radius from $K$ (or $K^{\prime}$ in the case of $\operatorname{arc} 2^{\prime}$ ) is aligned with $\mathbf{A}_{1}$. Consequently, the predicted illusion angle for arc 2 is $A_{1} \hat{R A} A_{2}$, while that for arc $2^{\prime}$ is $A_{1} \hat{R}^{\prime} A_{2}$ '. It will be seen that this angle is 0 deg if the central axis angle is $0 \mathrm{deg}$, and then increases as the central axis angle increases, reaching a maximum when the midpoint of arc 2 is at the same horizontal level as $A_{1}$. (The geometrical proof is given in Appendix 2.) Within this range, then, the prediction is that an illusion angle varies directly with the central axis angle.

Distance between the arcs. Figure $6 \mathrm{~b}$ shows the predicted effect of increasing the distance between the arcs while keeping the other parameters constant. Arc 1' represents the result of increasing the distance between arc 1 and arc 2 . It will be seen that the apparent center of arc $1^{\prime}$ is closer to $R$ than the apparent center of arc 1 , since arc 1 is shorter and its radius is thus overestimated by a greater magnitude. As before, the predicted value of the rotation angle is that which aligns the radius from $K$ with $\mathbf{A}_{1}$ (or $\mathbf{A}_{1}{ }^{\prime}$ in the case of arc $1^{\prime}$ ). Consequently, the predicted result is that: as the distance between the arcs increases, the illusion angle will decrease; the two variables are inversely proportional.

Curvature of the arcs. Since the overestimation of a radius is an inverse function of its length (Virsu, 1971a, 1971b; Micella, Note 2), the distance between the real and the perceived centers is smaller for arcs of lesser curvature. As a consequence, the predicted effect of increasing the radii of both arcs is to decrease the illusion angle.

Central angle of the arcs. As the central angle of the arcs increases, the predicted value of the illusion angle decreases, since the effect of the increase is to

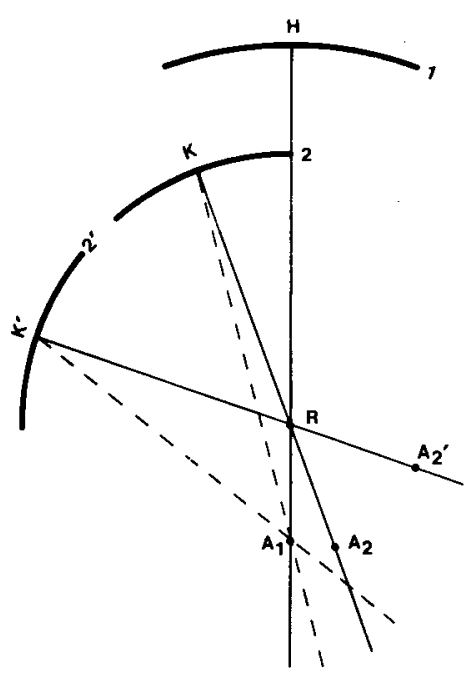

(a)

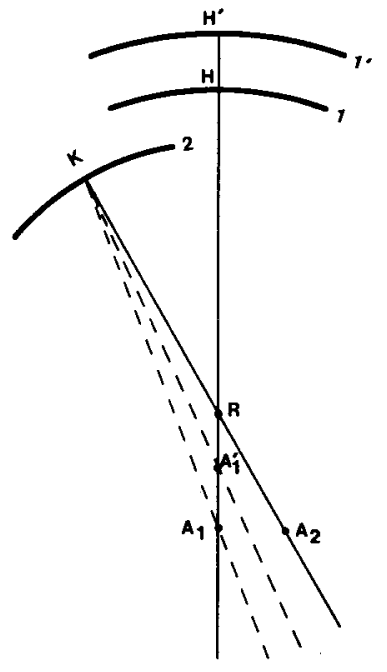

(b)

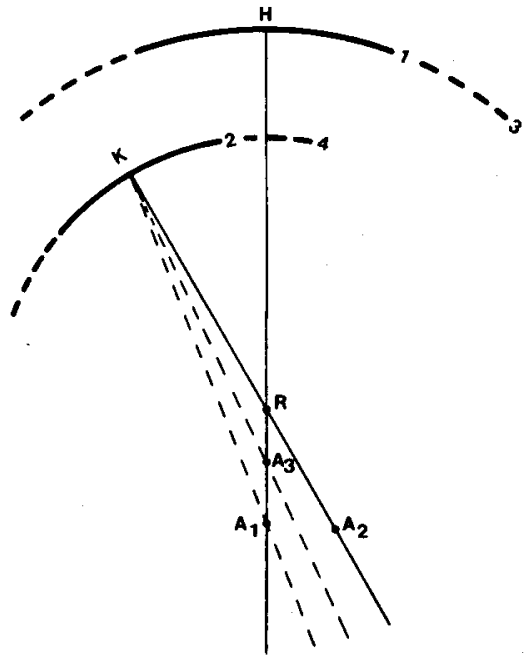

(c)

Figure 6. A graphic representation of an arc adjustment which serves to minimize the distance between the apparent centers, $A_{1}$ and $A_{2}$. Solid straight lines represent central axes of real concentric arcs. Dashed straight lines represent the axis of the lower arc 2 (or 4) after it has been reoriented in position to pass through $A_{1}$; by this adjustment the distance between $A_{1}$ and $A_{2}$ has the lowest value. In (c), the dashed curves stand for two arcs ( 3 and 4 ) that are longer than the solid arcs. Their apparent centers are indicated by the symbols $A_{3}$ and $A_{4}$. The differences between real and apparent centers are exaggerated for purposes of ilustration. 
Table 1

The Variations of Eccentricity Illusion Predicted by the Hypotheses Discussed in the Text

\begin{tabular}{|c|c|c|c|}
\hline \multirow{2}{*}{$\begin{array}{l}\text { Experimental } \\
\text { Manipulation }\end{array}$} & \multicolumn{3}{|c|}{ Hypotheses } \\
\hline & End Tangent & Centers Attraction & Averaging \\
\hline $\begin{array}{l}\text { Increase Central } \\
\text { Axis Angle }\end{array}$ & $\begin{array}{l}\text { Illusion Angle Increases } \\
\text { by an Equal Amount }\end{array}$ & $\begin{array}{l}\text { Illusion Angle Increases } \\
\text { by a Smaller Amount }\end{array}$ & Intermediate \\
\hline $\begin{array}{l}\text { Increase Distance } \\
\text { Between Arcs }\end{array}$ & No Change & $\begin{array}{l}\text { Illusion Angle } \\
\text { Decreases }\end{array}$ & Intermediate \\
\hline $\begin{array}{l}\text { Increase Radius } \\
\text { of Lower Arc }\end{array}$ & No Change & $\begin{array}{l}\text { Illusion Angle } \\
\text { Decreases }\end{array}$ & Intermediate \\
\hline $\begin{array}{l}\text { Increase Center } \\
\text { Angle of Arcs }\end{array}$ & No Change & $\begin{array}{l}\text { Illusion Angle } \\
\text { Decreases }\end{array}$ & Intermediate \\
\hline
\end{tabular}

bring the apparent radius of the upper arc closer to $R$ (see Figure 6c). A summary of the predictions is given in Table 1.

\section{METHOD}

Each stimulus consisted of two arcs (as in Figure 3). The center angles of the arcs were always equal, but in most cases the arcs were drawn in different circular sectors. The upper arc, $\widehat{\mathbf{A B}}$, was always placed in such a way that its central axis ran vertically down the center of the paper, whereas the lower arc, $\overline{C D}$, was presented at varying orientations, obtained by rotating it clockwise and anticlockwise about its midpoint, $\mathbf{K}$. The general method employed was to determine, using the method of limits, the orientation of CD (i.e., the value of the rotation angle) for which the arcs were perceived to be concentric. This value, which represents a measure of the extent of the illusion, will be called, as before, the "illusion angle." In precise terms, the illusion angle is the angular distance between real and perceived concentricity, clockwise deviations being (arbitrarily) regarded as positive. The aim of the experiment was to determine the effect on the illusion angle of variations in the following parameters: (1) the central axis angle, (2) the difference in length between the arc radii, (3) the curvature of the arcs, and (4) the center angle of the arcs.

\section{Materials}

Twenty-one series of stimuli were prepared, each series being based on a different configuration of the four parameters mentioned above. The stimuli within any given series differed from one another only with respect to the orientation of the arc $\overparen{C D}$, which was varied in small steps in order to produce a range of stimuli that could be used as either an ascending or a descending series. The parameter configurations were determined by selecting "standard" configuration and then varying each of its parameters independently. The standard configuration was: (1) central axis angle, 20 deg; (2) distance between arcs, $2 \mathrm{~cm}$; (3) length of radius of the lower arc, $6 \mathrm{~cm}$; and (4) center angle of the arcs, $40 \mathrm{deg}$. The alternative settings of each variable are shown in Table 2.

The number of stimuli in a series varied from 10 to 20 . Within each series, the rotation angle (i.e., the orientation of $\mathrm{CD}$ ) was varied in steps ranging from 1 to 4 deg. The overall range of the rotation angle was -20 to $50 \mathrm{deg}$, but, for any given series of stimuli, the difference between the highest and lowest value was only on the order of 30-40 deg.

Each stimulus was drawn on a white $20 \times 14 \mathrm{~cm}$ card in black india ink using a constant line thickness of $.05 \mathrm{~cm}$. The upper arc was always drawn at a distance of $3 \mathrm{~cm}$ from the top of the card, with its concave side facing downwards and with its central axis running vertically down the middle. The lower arc was drawn either on the lower left of the upper arc or (in one condition) directly below it.

\section{Subjects}

There were 20 subjects, 12 women and 8 men.

\section{Procedure}

The subject was seated in front of a low table. The stimuli were placed one by one on the table, and viewed vertically at a distance of about $40 \mathrm{~cm}$. Before the session began, the subject was asked what he understood by the word "concentric," and if necessary the concept was explained to him. The task was to respond, in each case, "convergent," "divergent," or "concentric," according to whether the right-hand ends of the arcs were perceived as converging, diverging, or concentric. The subjects were not permitted to move their hands or to alter the position of the stimulus.

The 18 series of stimuli were presented in random order, with each series being presented four times, twice ascending and twice descending. In this way, four estimates of the illusion angle were obtained for each condition. The order of the ascending (A) and descending (D) series for a given condition was either ADAD or DADA, the choice between these being random. The average length of the sessions was $60 \mathrm{~min}$, and, since the task was exhausting, subjects were told to ask for rest intervals when they felt tired. Each of the subjects participated in a single session.

\section{RESULTS}

Mean values of the illusion angle as a function of the four independent variables are shown in Fig-

Table 2

Magnitude of the Levels of Each Dimension for Every Stimulus Set

\begin{tabular}{cllll}
$\begin{array}{c}\text { Stimulus } \\
\text { Set }\end{array}$ & \multicolumn{1}{c}{$\begin{array}{c}\text { Center Angle } \\
(\gamma)\end{array}$} & \multicolumn{1}{c}{$\begin{array}{c}\text { Radii } \\
\text { Difference } \\
(\mathrm{R}-\mathrm{r})\end{array}$} \\
\hline 1 & $40 \mathrm{deg}$ & $0,10,20,30,40,50 \mathrm{deg}$ & 6 & 2 \\
2 & $40 \mathrm{deg}$ & $20 \mathrm{deg}$ & 6 & $1,2,3,4,5$ \\
3 & $40 \mathrm{deg}$ & $20 \mathrm{deg}$ & $4,5,6,7,8$ & 2 \\
4 & $40,50,60,70,80 \mathrm{deg}$ & $20 \mathrm{deg}$ & 6 & 2 \\
\hline
\end{tabular}

Note $-R$ and $r$ represent the radius of the top and of the lower arc, respectively. $\alpha$ is the chord interception angle; $\gamma$ is the arc center angle. 

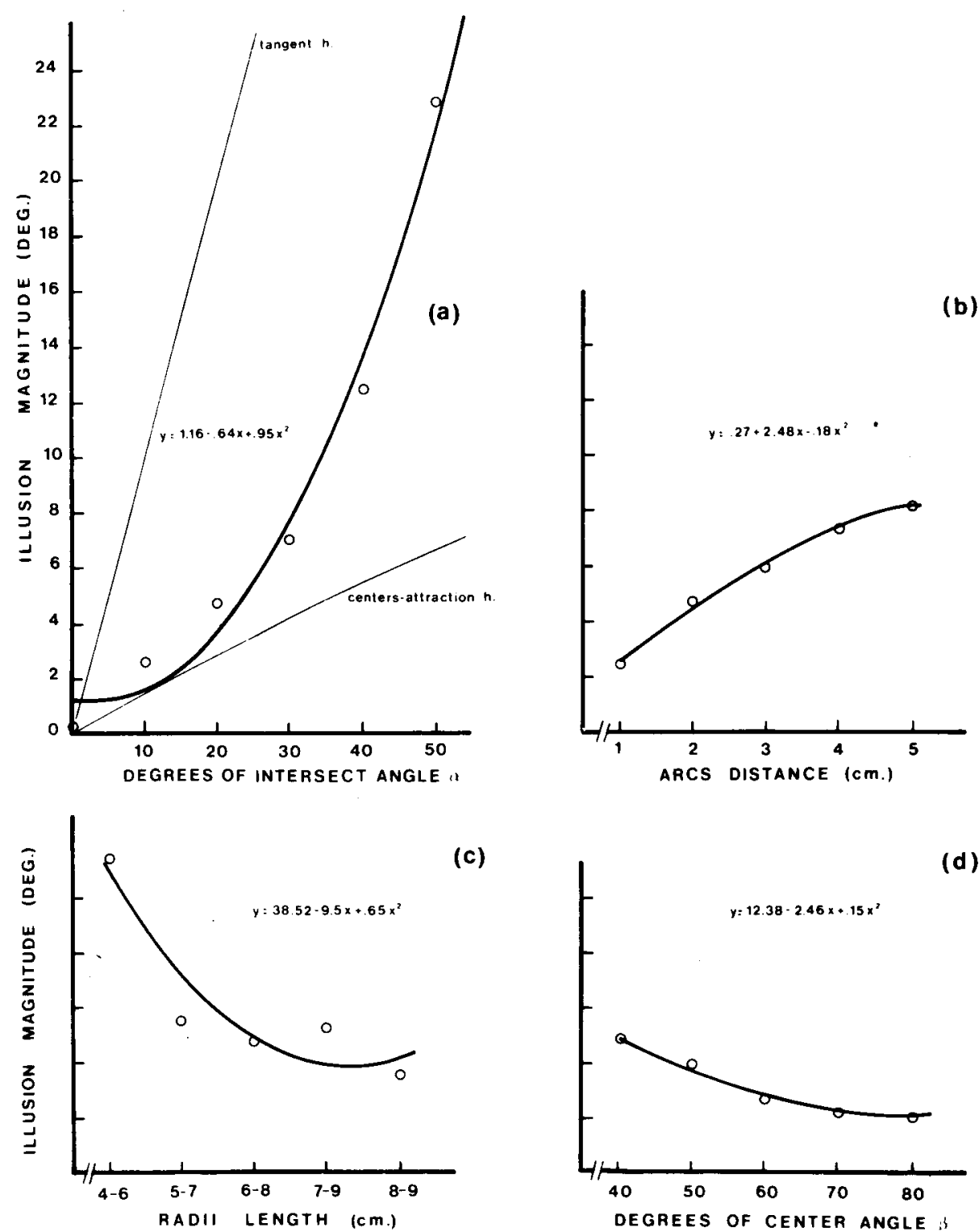

(c)

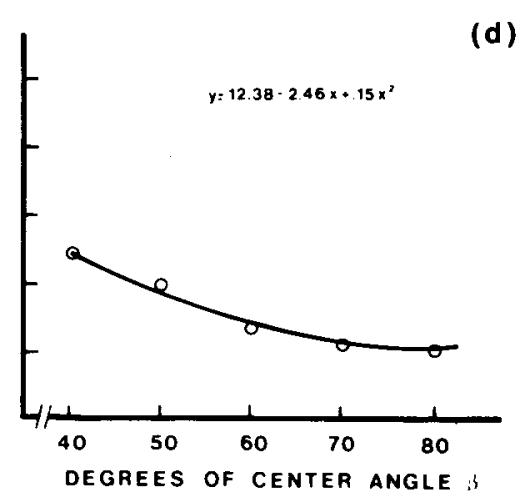

Figare 7: Open circles represent the mean angles of tilt (illusion magnitude) required to perceive the arcs as concentric. (a) The amount of illualon as a function of $a$ along with the values predicted by the end-tangent hypotheals and the centers-attraction hypotheals (oharp lines). The latter function was determined on the bash of Viru's (1971a) data. (b-d) The Illudion magnitudes as a function of arcs distance, arce radil, and length, respectively. The equations in the figures refer to the curve (the thick curve in the graph) best nitting the results. Goodness of $\mathrm{fit}$, in pereent, resulted in 99.9 for the data In (a), 99.7 for those in (b), and 95 and 99.7 for those shown in (c) and (d), reapectlvely.

ures $7 \mathrm{a}-7 \mathrm{~d}$. It will be seen that all these mean values are positive, indicating that the direction of the illusion was consistently clockwise. (This, of course, is because the lower arc is to the left of the upper arc; if it had been to the right, the direction of the illusion would have been anticlockwise.) The detailed results for each of the four variables are given below.

\section{Central Axis (Figure 7a)}

The illusion angle increased sharply as the central axis angle was increased. A one-way analysis of vari- ance conducted on the data for this variable showed that the effect highly significant $[F(5,95)=211.59$, $p<.001]$. Figure $7 \mathrm{a}$ suggests that the curve of the best fit is quadratic rather than linear; this was confirmed by a trend test which showed a significant quadratic trend $[F(1,95)=105.93, p<.001]$.

\section{Difference Between the Lengths of the Radil (Figure 7b)}

The illusion angle increased as the difference between the radius lengths was increased. The effect was significant $[F(4,76)=79.27, p<.001]$. 


\section{Length of the Radius of Arcs}

The illusion angle decreased as the radius length was increased $[F(4,76)=41.47, p<.001]$.

\section{Center Angle of the Arcs}

The illusion angle decreased as the center angle was increased $[F(4,76)=41.47, p<.001]$.

\section{DISCUSSION}

The results did not consistently confirm any of the hypotheses. The end-tangent hypothesis (which predicts that the value of the illusion angle is determined by the central axis angle) is refuted by the results of Figures $7 \mathrm{~b}-7 \mathrm{~d}$, which show that each of the other three variables examined influences the extent of the illusion: according to the end-tangent hypothesis, the value of the illusion angle in these three conditions should be constant. The shape of the curve in Figure $7 \mathrm{a}$ is also different from that predicted, particularly in the lower range (i.e., for lower values of the central axis angle).

The centers-attraction hypothesis correctly predicts that decline in the value of the illusion angle in Figures $7 \mathrm{c}$ and $7 \mathrm{~d}$, and also gives a reasonable approximation to the results in the lower range of Figure 7a. It is refuted, however, by the results of Figure $7 b$, which shows that an increase in $R-r$, the difference between the radius lengths, does not decrease the extent of the illusion (as predicted), but, rather, increases it. The hypothesis also fails to account for the sharp rise in the value of the illusion angle in the upper range of Figure $7 \mathrm{a}$ (i.e., the range in which the central axis angle increases from 30 to $50 \mathrm{deg}$ ).

The averaging hypothesis receives some support from Figures $7 \mathrm{a}, 7 \mathrm{c}$, and $7 \mathrm{~d}$, but, like the centersattraction hypothesis, it is refuted by Figure $7 \mathrm{~b}$.

Reassessing the situation in the light of these results, the following conclusions may be drawn. (1) There is evidence that both of the hypothesized distortion mechanisms play some part in the illusion. The effect of the end-tangent mechanism is seen in the upper range of Figure 7a, and the effect of the center-attraction mechanism in Figures $7 \mathrm{c}$ and $7 \mathrm{~d}$ and in the lower range of Figure 7a. (2) The shape of the curve in Figure 7a suggests that the relative importance of the two distortion mechanisms varies with the central axis angle. The equations proposed by Coren and Ward (1979),

$$
\begin{gathered}
W_{i} I_{i}+W_{j} I_{j}=I_{i} \cup I_{j} \\
W_{i}, W_{j}>0 \\
W_{i}+W_{j}=1,
\end{gathered}
$$

allow us to estimate the weighting factors for each value of the central axis angle considered here by sub-
Table 3

Estimates of the Weighting Factors: Tendency to Adjust the

Tangents $\left(W_{i}\right)$ and Tendency to Approach the Centers $\left(W_{j}\right)$

\begin{tabular}{ccccccc} 
& \multicolumn{5}{c}{$\alpha$} \\
\cline { 2 - 6 } & $0 \mathrm{deg}$ & $10 \mathrm{deg}$ & $20 \mathrm{deg}$ & $30 \mathrm{deg}$ & $40 \mathrm{deg}$ & $50 \mathrm{deg}$ \\
\hline $\mathrm{W}_{\mathrm{i}}$ & & .14 & .11 & .11 & .20 & .37 \\
$\mathrm{~W}_{\mathrm{j}}$ & .86 & .89 & .89 & .80 & .63 \\
\hline
\end{tabular}

stituting for $I_{j}$ the value predicted by Equation 1, for $I_{i}$ the magnitudes of the central axis angle, and for $I_{i} \cup I_{j}$ the mean estimates of the illusion here observed. The coefficients thus found are given in Table 3. As one can see, the tendency to approach the perceived centers is always the strongest, but it decreases in the range $40-50 \mathrm{deg}$ of the central axis angle. (3) The results depicted in Figure $7 b$ (the $R-r$ condition) cannot be attributed to any combination of the two mechanisms and thus suggest that some other mechanism must be taken into account. In sum, what seems to be needed is a more complex model in which the extent of the illusion depends on a weighted combination of the end-tangent mechanism, the centers-attraction mechanism, and some other mechanism that would explain the Figure $7 \mathrm{~b}$ results. Such a model will now be proposed

\section{The Variable-Weights Hypothesis}

Until now, it has been assumed that concentricity judgments are based on two types of test: (1) a test of the parallelism of the arcs, and (2) a test of whether the centers of the arcs coincide. It has further been assumed that the parallelism test is carried out by mentally elongating the arcs and determining whether they converge; this method, as we have seen, produces a distortion owing to the tendency to follow the end tangents when attempting to elongate the arcs.

A possibility that has not been considered is that the parallelism test is carried out by another method: namely, by measuring at several points the distance between those parts of the two arcs that overlap. This will henceforth be called the "equidistance" method. The crucial difference between the equidistance method and the end-tangent method is that the former involves no elongation of the arc and consequently introduces no distortion. It is therefore a reasonable conjecture that variations in the extent of the illusion may be due to whether or not circumstances favor the use of the equidistance method. If the disposition of the arcs favors this method, the illusion should be slight; if, instead, it favors the endtangent method, the illusion should be greater. The ideal conditions for applying the equidistance method are shown in Figure 8a. Here, the arcs overlap to the maximum possible extent, and the distance between them is relatively small: consequently, the distance between the arcs can be measured at a wide 

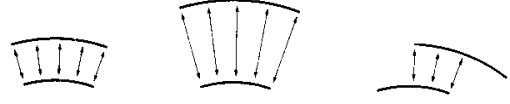

(b)

(c)

Figure 8. A schematic illustration of a test of arc equidistance. The arrows indicate some imaginary segments that an observer takes as a measure of the distance of corresponding points (the points lying upon the same radius) of the arcs.

range of points and with relatively high accuracy. In Figure 8c, instead, the overlap is slight and the range of possible measures consequently much smaller, while in Figure $8 b$ the arcs are relatively far apart and the distance measures are less accurate. It may be predicted that there will be a greater tendency in such cases as Figures $8 \mathrm{~b}$ and $8 \mathrm{c}$ to rely on the end-tangents method. In general, the importance of the endtangents method as compared with the equidistance method should increase (1) as the central axis angle increases (since this reduces overlap), and (2) as the distance between the arcs increases.

The more complex model resulting from the introduction of the equidistance test may be represented as follows:

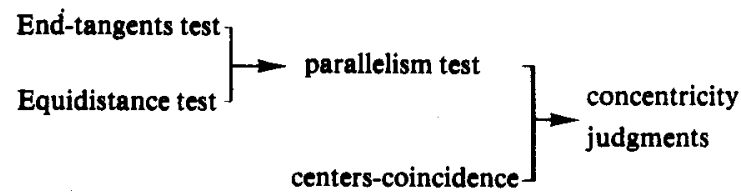

test

It will be assumed that the weights attached to the parallelism and the centers coincidence tests are invariant, while within the parallelism test, instead, the relative weight of the equidistance and endtangent tests vary according to the value of the central axis angle and the distance between the arcs.

Explanations can now be given for the two problematic results mentioned above. First, of all, the tendency for the illusion angle to increase as the distance between the arcs (i.e., $R-r$ ) is lengthened can be explained by a change in the relative weights of the end-tangent test and the equidistance test. For any constant value of the central axis angle, the weight given to the equidistance test will decline as the distance between the arcs is lengthened, while the weight given to the end-tangent test will correspondingly increase. The more weight given to the endtangent test, the more distortion will be introduced. Consequently, as the distance $R-r$ is increased, the distortion introduced by the parallelism test will increase, while, as we have seen, the distortion due to the centers-coincidence test will decline. The outcome will thus depend on which distortion is greater: assuming that the distortion due to the end-tangent effect predominates, the predicted result will be as in Figure $7 b$.
A similar argument can explain the other problematic result, the shape of the curve in Figure 7a. As the central axis angle increases, the end-tangent test should assume greater importance relative to the equidistance test, and the distortion due to the parallelism test should thus increase. Consequently, for low values of the central axis angle, the distortion should be primarily due to the centers-attraction mechanism, while for the higher values of the central axis angle, the end-tangents mechanism should come increasingly into play and the increase in the illusion angle should accelerate, just as occurs in Figure 7a.

The variable-weights hypothesis states that the eccentricity illusion depends on the interaction of several distorting mechanisms. Other recent theories of visual geometrical illusion have also postulated explanations of this sort (Coren, Girgus, Erlichman, \& Hakstian, 1976; Coren \& Ward, 1979); in particular, Virsu's (1971a, 1971b) work on the perception of curvature takes into account several mechanisms similar to those considered here. Nevertheless, in order to justify the complexity of the proposed theory, I shall conclude by attempting to demonstrate as clearly as possible the relevance of each of the postulated distortion mechanisms and the possibility of reducing, by their action some of the known misperceptions of curvature.

The relevance of the end-tangent mechanism is intuitively apparent from Figures $9 a$ and $9 b$. Figure $9 a$ shows two nonconcentric arcs, drawn in such a way that their end tangents obviously converge; the result is a clear perception of eccentricity. Figure $9 \mathrm{~b}$ shows exactly the same arcs but in a different arrangement so that the end-tangent crossing is not easily determinable; the result is that their eccentricity is no longer obvious and they might, indeed, be judged concentric. The relevance of the centers-attraction mechanism is brought out by Figure 9c. In this figure, the arcs have been drawn in such a way that their end tangents are parallel; nevertheless, because of the obvious separation of their centers, they are perceived as eccentric. Also, the arcs in Figure 9d are drawn so that the end tangents are parallel, but this configuration represents a more interesting case. The arcs are perceived as converging on both sides if the impression is of a series of curves in a plane. But

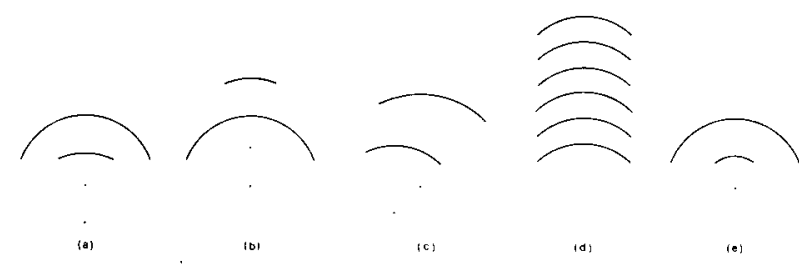

Figure 9. (a, b, d) Arcs of the same curvature but with different locations of centers. (c) Arcs with different curvature and radius but with parallel tangents to the end points. (e) Two concentric arca of different radius. The points, when drawn, represent the locatons of the centers. 
when the arcs are perceived as sections of parallel disks, a condition in which the tangents to one arc cannot intercept the tangents to the other, the impression of convergence vanishes and the arcs appear as to be drawn on different parallel sheets. It is probably this illusion of depth that underlies the widening of the top of configurations in Figure 9d that was observed by Wundt (1898) (quoted by Robinson, 1972).

Figure 9e shows the flattening of the shorter of two concentric arcs. As with Figures 1 and 2, this last configuration demonstrates that even the coincidence of centers cannot ensure the correct perception of concentricity.

Thus, our conclusion is that the perception of concentricity requires two conditions: the end-tangents parallelism and the centers coincidence. When only one is satisfied, as in Figures 1c, 2, and 3a, a particular sort of concentricity can be reconstructed by reorienting one of the two arcs, such as concentricity resulting from the balance of two opposing tendencies.

\section{REFERENCE NOTES}

1. Roncato, $S$. Indagine preliminare su un'illusione di eccentricita (Report No. 1). Padua, Italy: Istituto di Psicologia, 1980.

2. Micella, F. Misperception of curvature as a function of radii and arcs length. Unpublished mimeographed manuscript, University of Padua, Italy, 1982.

\section{REFERENCES}

Coren, S., Gingus, J. S., Enlichyan, H., \& Haketian, A. R. An empirical taxonomy of visual illusions. Perception \& Psycho. physics, 1976, $20,129-137$.

Coren, S., Ward, L. M. Levels of processing in visual illusions: The combination and interaction of distortion-producing mechanisms. Journal of Experimental Psychology: Human Per. ception and Performance, 1979, 5, 324-334.

Crassini, B., \& Oven, R. Musking, after-effect and illusion in the visual perception of curvature. Perception \& Psychophysics, $1975,17,411-416$.

Fraser, J. A new visual illusion of direction. British Journal of Psychology, 1908, 2, 307-320.

Robinson, J. O. The psychology of visual illusion. London: Hutchinson, 1972.

Timney, B. N., \& McDonatd, C. Are curves detected by 'curvature detectors'? Perception, 1978, 7, 51-64.

Tolansxy, S. Optical Illusions. Oxford: Pergamon Press, 1964.

VIrsu, V. Tendencies to eye movement, and misperception of curvature, direction and length. Perception \& Psychophysics, 1971, 9, 339-342. (a)

VIrsu, V. Underestimation of curvature and task dependence in visual perception. Perception and Psychophysics, 1971, 9, 339-342. (b)

Wundr, W. Die geometrisch-optischen Taeuschungen. Akedemie der Saechs, Wissenschaften, Leipzig-Abnandlungen, 1898, 24, 53-178.

\section{APPENDIX 1}

In Figure 6a, the rotation angle of arc 2 that minimizes the distance between the perceived centers $\mathbf{A}_{1}$ and $\mathbf{A}_{\mathbf{2}}$ of arcs 1 and 2 is $R \widehat{K} A_{1}$. This can be proved by showing that when the line $\mathbf{K A}_{\mathbf{2}}$ is rotated about $K$, the minimal distance between $A_{1}$ and $A_{2}$ is obtained when $K A_{2}$ passes through $A_{1}$. In Figure 10a, the circle represents the possible positions
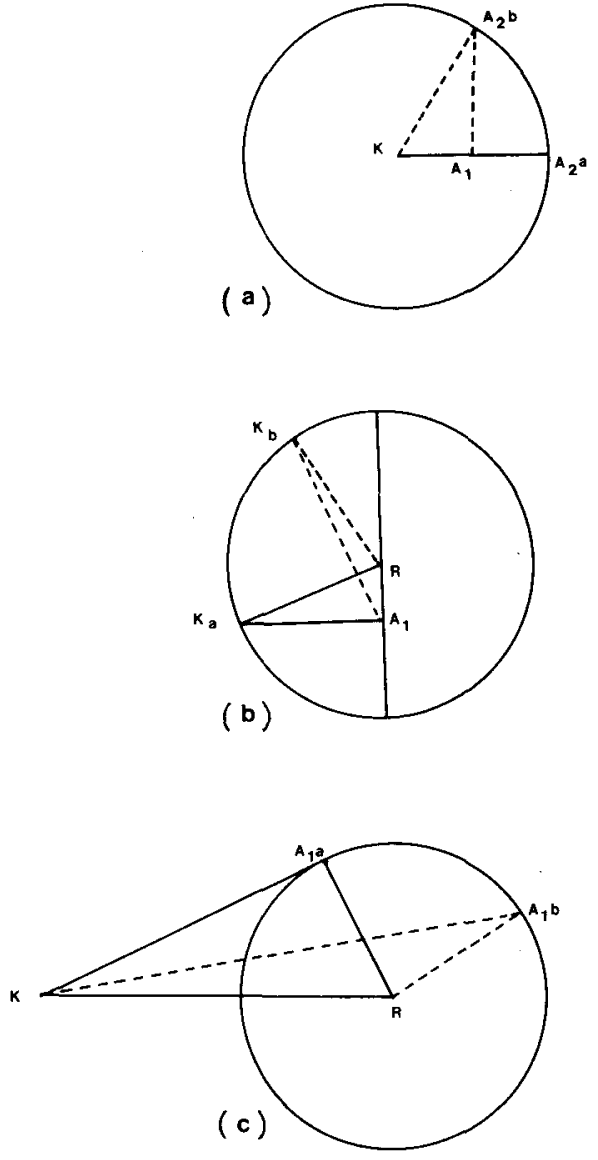

Figure 10. Methods of demonstrating the conditions in which, according to the centern-attraction hypothesis, (a) the distance between the apparent centers is the shortest one, and (b) and (c) the illusion angle reaches the highest value. See text for discussion.

of $A_{2}$ if $K A_{2}$ is rotated about $K ; A_{2} a$ is that position of $A_{2}$ for which $K A_{2}$ passes through $A_{1}$, and $A_{2} b$ is another arbitrary position for which $\mathrm{KA}_{2}$ does not pass through $\mathbf{A}_{1}$. The problem is thus to prove that the line $A_{1} A_{2} b$ is longer than $A_{1} A_{2} a$. Since $K A_{1} A_{2} b$ is a triangle,

$$
K A_{1}+A_{1} A_{2} b>K A_{2} b \text {. }
$$

But since $\mathrm{KA}_{2} \mathrm{~b}$ is a radius of the circle,

$$
K A_{2} b=K A_{2} a=K A_{1}+A_{1} A_{2} a \text {. }
$$

Therefore,

$$
K A_{1}+A_{1} A_{2} b>K A_{1}+A_{1} A_{2} a_{1}
$$

and therefore,

$$
A_{1} A_{2} b>A_{1} A_{2} a \text {. }
$$

\section{APPENDIX 2}

In Figure 6a, as the center $K$ of arc 2 is rotated about $R$, the maximum value of the angle $R \widehat{K} A_{1}$ is obtained when the angle $K \widehat{A}_{1} R$ is 90 deg. This may be proved as follows. 
In Figure 10b, the circle represents possible positions of $K$ if $R K$ is rotated about $R$. Ka is a position of $K$ for which $K A_{1} R$ is a right angle, and $K b$ is an arbitrary position of $K$ for which $K_{1} R$ is not a right angle. The problem is to prove that angle $\mathrm{RKaA}_{\mathbf{1}}>\mathrm{RKbA}_{\mathbf{1}}$. Now, the set of triangles $R A_{1} K$ that we obtain when we rotate $K R$ about $R$ may also be represented as in Figure 10c. This diagram represents the results of rotating all triangles $R A_{1} K$ about $R$ so that the sides $R K a, R K b, R K c$, etc., are all aligned; the point $A_{1}$ now varies, and can take any of the positions in- dicated by the circle in Figure 10c. The values of $A_{1}$ that are marked, namely $A_{1} a$ and $A_{1} b$, are such that triangle $R \widehat{A}_{1} a K$ is congruent with $R \widehat{A}_{1} \mathrm{Ka}$, and triangle $R \widehat{A}_{1} b K$ is congruent with $R A_{1} K b$. It is now evident that the maximum value of the angle $A_{1} \widehat{K} R$ is obtained when $K A_{1}$ is a tangent to the circle: that is, when angle $K A_{1} R$ is $90 \mathrm{deg}$.

(Manuscript received February 19, 1982;

revision accepted for publication September 28, 1982.) 